\title{
Time Resolved Magnetization Dynamics of Ultrathin Fe(001) Films: Spin-Pumping and Two-Magnon Scattering
}

\author{
G. Woltersdorf, ${ }^{1,2}$ M. Buess, ${ }^{2}$ B. Heinrich, ${ }^{1}$ and C. H. Back ${ }^{2}$ \\ ${ }^{1}$ Simon Fraser University, 8888 University Drive, V5A 1S6 Burnaby BC, Canada \\ ${ }^{2}$ Universität Regensburg, Universitätsstraße 31, 93040 Regensburg, Germany
}

(Received 19 November 2004; published 11 July 2005)

\begin{abstract}
The time-resolved magnetic response of ultrathin epitaxial $\mathrm{Fe}(001)$ films grown on $\mathrm{GaAs}(001)$ and covered by $\mathrm{Au}, \mathrm{Pd}$, and $\mathrm{Cr}$ capping layers was investigated by time and spatially resolved Kerr effect measurements. The magnetization was excited by an in-plane magnetic field pulse using the transient internal field generated at a Schottky barrier while the wavelength of the excitation (resonant mode) was roughly $4 \mu \mathrm{m}$. Each of the three cap layers affected the spin relaxation in a unique way. Au cap layers resulted in the bulk Gilbert damping of the Fe film. Pd cap layers caused an additional Gilbert damping due to spin-pump or spin-sink effects. Cr cap layers lead to a strong extrinsic damping which can be described by two-magnon scattering. In this case the strength of the extrinsic damping can be controlled by a field induced shift of the spin wave manifold with respect to the excited $k$ vector.
\end{abstract}

PACS numbers: 78.20.Ls, 75.30.-m

Ferromagnetic relaxation is intensely studied because it is of utmost importance for high frequency spintronics applications. A typical example is the read sensor in magnetic recording heads. Here, the figure of merit is limited by thermal noise and the damping parameter of the magnetic thin films used in these devices [1]. Thus, in order to control the magnetization dynamics and the noise performance of such devices it is essential to understand and control the spin relaxation processes. A significant portion of the ferromagnetic relaxation can be due to the generation of spin waves. This process can be described by modemode coupling [2]. Time-resolved Kerr microscopy (TRMOKE) is a technique which allows one to locally probe the time evolution of the magnetization and therefore allows one to study the spin dynamics in small geometries. In this Letter, we will consider the time-resolved motion of the magnetization in ultrathin epitaxial Fe films having different metallic cap layers. These capping layers are $\mathrm{Au}, \mathrm{Pd}$, and $\mathrm{Cr}$. We will show that the choice of the capping layer dramatically alters the spin dynamics of the underlying ultrathin Fe film. In the ferromagnetic resonance (FMR) measurements the resonance mode was spatially homogeneous and the $k$ vector of the excitation close to zero. In contrast to FMR the TRMOKE measurements allow one to study the spin dynamics on a micrometer scale and at nonzero $k$ vectors. We examine how different relaxation mechanisms manifest themselves on micrometer length scales corresponding to the lateral geometries which are important for device applications.

The time-resolved motion of the magnetization was measured in response to a small ultrashort magnetic field pulse generated by the current from the illuminated Schottky diode at the $\mathrm{Fe} / \mathrm{GaAs}(001)$ interface [3]. The polar magnetization component was monitored using TRMOKE. $\mathrm{Au}, \mathrm{Pd}$, and $\mathrm{Cr}$ metallic layers were used to cover a 16 monolayer (ML) thick Fe film.
The metallic multilayers were grown by molecular beam epitaxy (MBE) on GaAs(001) wafers (see details in [4,5], and references therein). An ohmic contact was formed at the back side of the wafer, and a bias voltage of $-2 \mathrm{~V}$ was applied between the GaAs substrate and the metallic film [see Fig. 1(a)]. TRMOKE was measured by a pump-probe technique. Ultrashort light pulses $(\sim 150 \mathrm{fs})$ are generated by a $800 \mathrm{~nm}$ Ti:sapphire laser system and used to (i) tip the magnetization vector out of equilibrium (pump) by focusing the light onto the sample with $\sim 2 \mu \mathrm{m}$ spot diameter (details of the pumping mechanism are discussed below) and (ii) to probe the magnetization. The probe beam is frequency doubled and then focused by the same objective

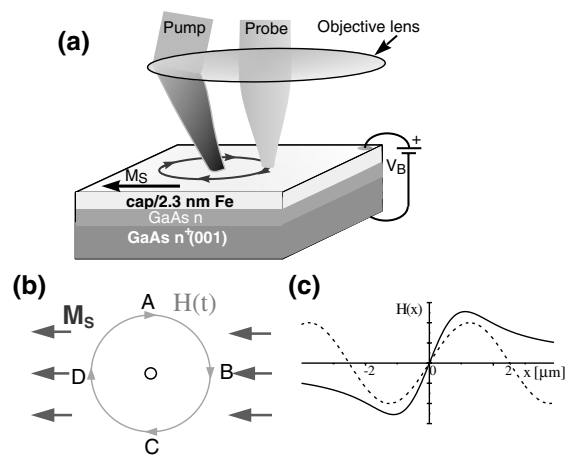

FIG. 1. (a) Cartoon of the experimental configuration for the TRMOKE measurements. Pump and probe beams were focused by the same objective lens and are offset by roughly $1 \mu \mathrm{m}$. (b) Plan view of the orientation of the circular magnetic field pulse $H(t)$ resulting from optical pumping at the center of the circle. The orientation of the saturation magnetization is shown by the bold arrows. (c) The graph on the right shows the spatial dependence of the magnetic pulse field amplitude $H(x)$ along the direction parallel to the magnetization. The dashed line represents the leading Fourier component of the magnetic response. 
lens with a spot size of $\leq 1 \mu \mathrm{m}$ to measure the polar Kerr effect.

Figure 1(a) shows a cartoon of the experimental configuration used for the TRMOKE experiments. The optical pump beam generates a photocurrent which flows perpendicular to the $\mathrm{Fe} / \mathrm{GaAs}$ interface; its corresponding magnetic field is oriented in the film plane with circular field lines centered on the spot of the pump beam [see Figs. 1(a) and 1(b)]. The direction of the saturation magnetization $\boldsymbol{M}_{S}$ is fixed by an external bias field, $H_{B}$, which is parallel with the magnetic easy direction of the sample. In the parts of the sample where the pump field direction is perpendicular to the magnetization, the excitation torque reaches a maximum [points $B$ and $D$ in Fig. 1(b)], and in places where $\boldsymbol{M}_{S}$ is parallel or antiparallel to the pump field, no excitation takes place [points $A$ and $C$ in Fig. 1(b)]. Since the pump beam and the corresponding photocurrent have a nearly Gaussian intensity profile, the field amplitude as a function of distance $x$ from the pump spot center can be approximated by

$$
H(x) \sim \frac{1}{x}\left(1-e^{-x^{2} / R^{2}}\right),
$$

where $R$ is the radius of the laser pump spot. This function is antisymmetric and shown in Fig. 1(c).

Figure 2(a) shows the measured time evolution of the perpendicular component of the magnetization in a $20 \mathrm{Au} / 16 \mathrm{Fe} / \mathrm{GaAs}^{n} / \mathrm{GaAs}^{n+}$ sample. The integers represent the number of monolayers. The magnetization was excited by the magnetic in-plane field associated with the photocurrent generated by the laser pump beam in the $\mathrm{Fe} / \mathrm{GaAs}$ Schottky barrier [3]. In addition to the magnetic field pulse, the specimens were subjected to a uniform dc bias field of variable strength, $H_{B}$, applied in the plane of the film along the magnetic easy axis. The 16 ML Fe films had a strong in-plane uniaxial anisotropy which was generated at the $\mathrm{Fe} / \mathrm{GaAs}(001)$ interface [4]. In response to the magnetic field pulse, the magnetization was triggered to a free ferromagnetic resonance precession resulting in an oscillatory TRMOKE signal.
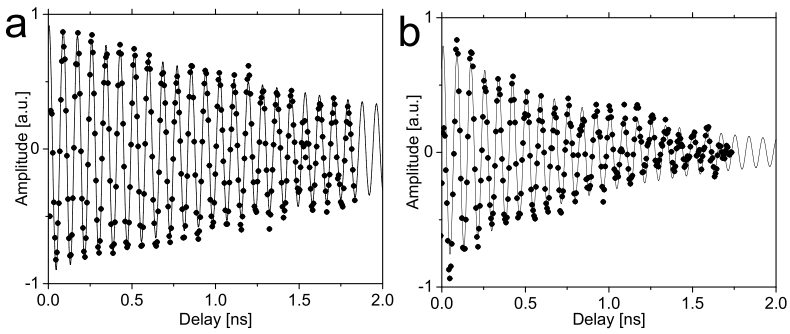

FIG. 2. Time-resolved magnetization data for (a) $20 \mathrm{Au} / 16 \mathrm{Fe} / \mathrm{GaAs}$ with the bias field $H_{B}=500 \mathrm{Oe} \|$ $[110]_{\mathrm{Fe}}$ leading to oscillations at $f=11.8 \mathrm{GH} z$ with a decay time $\tau=1.9 \mathrm{~ns}$ and (b) $20 \mathrm{Au} / 25 \mathrm{Pd} / 16 \mathrm{Fe} / \mathrm{GaAs}$ with $H_{B}=$ 500 Oe $\|[110]_{\mathrm{Fe}}(f=12.0 \mathrm{GH} z, \tau=1.1 \mathrm{~ns})$. The solid lines are fits obtained by using Eq. (2). The enhanced relaxation in the $20 \mathrm{Au} / 25 \mathrm{Pd} / 16 \mathrm{Fe} / \mathrm{GaAs}$ sample is caused by spin pumping.
The time evolution of the magnetization was measured for magnetic bias fields between 0 and $1 \mathrm{kOe} . H_{B}$ was applied parallel to the easy axis $\left([110]_{\mathrm{Fe}}\right)$. Figure 2 shows typical TRMOKE data for the $20 \mathrm{Au} / 16 \mathrm{Fe} / \mathrm{GaAs}$ and $20 \mathrm{Au} / 25 \mathrm{Pd} / 16 \mathrm{Fe} / \mathrm{GaAs}$ samples with $H_{B}=500 \mathrm{Oe}$. This bias field lead to a FMR frequency of $\sim 12 \mathrm{GHz}$. As expected for Gilbert damping in the limit $H_{B} \ll 4 \pi M_{\text {eff }}$, the decay time of the oscillations was found to be nearly independent of the bias field (see Fig. 4). The presence of a Pd cap layer decreased the decay time by almost $50 \%$.

The behavior was very different for the $\mathrm{Cr}$ covered $\mathrm{Fe}$ film. In Fig. 3 the data for the $20 \mathrm{Au} / 20 \mathrm{Cr} / 16 \mathrm{Fe} / \mathrm{GaAs}$ sample are shown for four different bias fields directed along the $[110]_{\mathrm{Fe}}$ direction. Clearly, the relaxation appears to be much faster compared to the samples with $\mathrm{Pd}$ and $\mathrm{Au}$ cap layers. However, under certain conditions $\left(H_{B}\right.$, pump beam focus) the damping was reduced by approximately $60 \%$, as shown in Figs. 3(d) and 5 (open star at $11.3 \mathrm{GHz}$ ). For $H_{B}=250$ Oe the FMR oscillations suddenly changed the phase by $180^{\circ}$, as illustrated in Fig. 3(b).

For homogeneous precession the magnetization dynamics can be analyzed by (i) numeric integration of the Landau-Lishitz-Gilbert equation (LLG) and (ii) analytic solution of the LLG which is valid for small angle motion. The amplitude, damping constant, and magnetic anisotropy fields are adjusted to achieve the best fit. Since the pump fields were only of the order of 20 Oe and the samples had in-plane anisotropy fields of the order of $\sim 500$ Oe, the precessional angle in these TRMOKE measurements was less than $0.5^{\circ}$. Therefore $m_{\mathrm{rf}} \ll M_{S}$, where $M_{S}$ is the saturation magnetization and $m_{\mathrm{rf}}$ the transverse rf component. In the limit of small damping $(\alpha \ll 1)$ one can
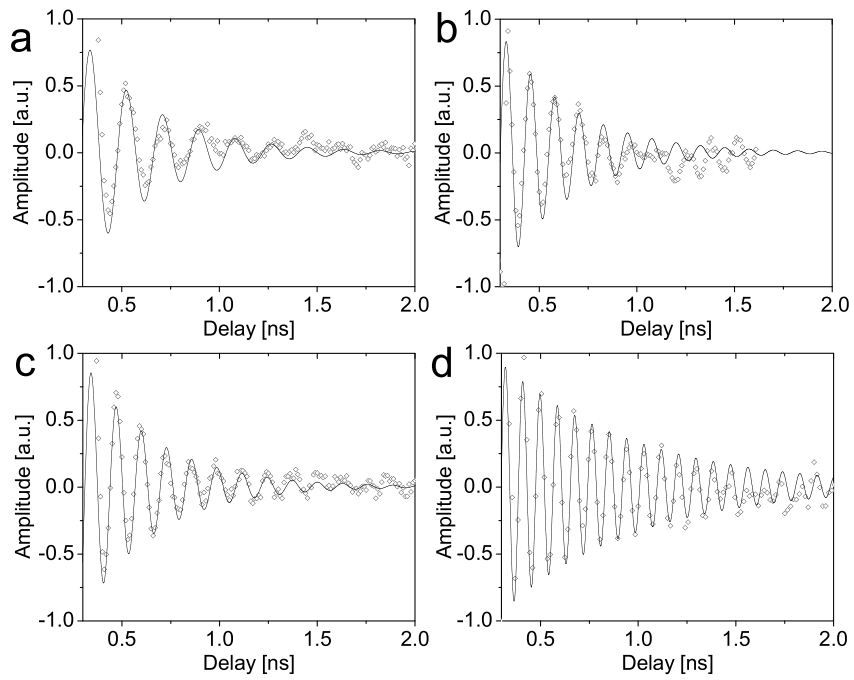

FIG. 3. Four data sets showing the time evolution of the magnetization in the $20 \mathrm{Au} / 20 \mathrm{Cr} / 16 \mathrm{Fe} / \mathrm{GaAs}$ sample with $H_{B} \|$ $[110]_{\mathrm{Fe}}$ at four different bias fields: (a) $H_{B}=0 \mathrm{Oe}(f=$ $6.1 \mathrm{GH} z, \tau=280 \mathrm{ps})$; (b) $H_{B}=250 \mathrm{Oe}(f=8.6 \mathrm{GH} z, \tau=$ $330 \mathrm{ps}) ; \quad$ (c) $H_{B}=500 \mathrm{Oe} \quad(f=9.4 \mathrm{GH} z, \quad \tau=330 \mathrm{ps})$; (d) $H_{B}=750 \mathrm{Oe}(f=11.3 \mathrm{GH} z, \tau=800 \mathrm{ps})$. Note that the damping in (d) is reduced by approximately $60 \%$. 


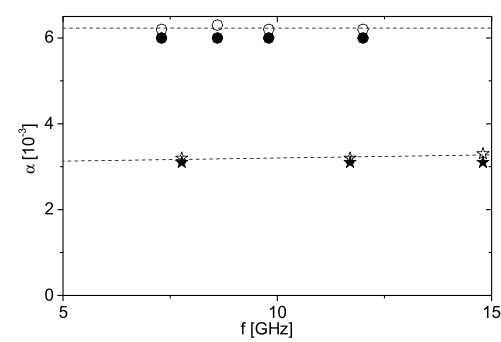

FIG. 4. The damping parameter $\alpha$ determined for different bias fields (frequencies) for the $20 \mathrm{Au} / 16 \mathrm{Fe} / \mathrm{GaAs}(\star t)$ ) and for the $20 \mathrm{Au} / 25 \mathrm{Pd} / 16 \mathrm{Fe} / \mathrm{GaAs}(\bigcirc)$ samples. The solid symbols are obtained from fits using numeric solutions of the LLG equations and the open symbols are obtained from fits using the analytic function described by Eq. (2).

write for the FMR precession

$$
m_{Z}(t)=m_{Z}(0) e^{-t / \tau} \cos (\omega t+\phi),
$$

where $\tau$ is the decay time $\tau=\frac{2}{\alpha \gamma\left(B_{\text {eff }}+H_{\text {eff }}\right)}$ and $\omega$ is the angular frequency of the free precession, $\omega=\gamma \sqrt{B_{\text {eff }} H_{\text {eff }}}$. $\alpha$ is the dimensionless Gilbert damping constant and $\gamma=$ $\frac{g|e|}{2 m c}$ is the absolute value of the spectroscopic splitting factor. $H_{\text {eff }}$ and $B_{\text {eff }}$ include the magnetic anisotropy and external fields and correspond to in-plane and out-of-plane stiffness fields, respectively [6]. For $H_{B}$ and $M_{S}$ parallel to the $[110]_{\mathrm{Fe}}$ direction, the in and out of plane stiffness fields are $B_{\mathrm{eff}}^{[110]}=H_{B}+4 \pi M_{\mathrm{eff}}+\frac{K_{1}^{\|}}{M_{S}}$ and $H_{\mathrm{eff}}^{[110]}=H_{B}-\frac{2 K_{1}^{\|}}{M_{S}}-$ $\frac{2 K_{U}^{\|}}{M_{S}}$, where $4 \pi M_{\text {eff }}=4 \pi M_{S}-\frac{2 K_{U}^{\perp}}{M_{S}}$ is the effective demagnetizing field. $K_{1}^{\|}$and $K_{U}^{\|}$are the in-plane fourfold and uniaxial anisotropy constants. For the $20 \mathrm{Au} / 16 \mathrm{Fe} / \mathrm{GaAs}$ sample the in-plane anisotropies were $K_{U}^{\|}=-5.5 \times$ $10^{5} \mathrm{erg} / \mathrm{cm}^{3}$ (hard axis $\|[1 \overline{1} 0]$ ) and $K_{1}^{\|}=2.3 \times$ $10^{5} \mathrm{erg} / \mathrm{cm}^{3}$ [4].

Fits to the data using Eq. (2) and numeric solutions of the LLG describe the data well. The results of the analyses (analytic and numeric) are identical and the damping pa-

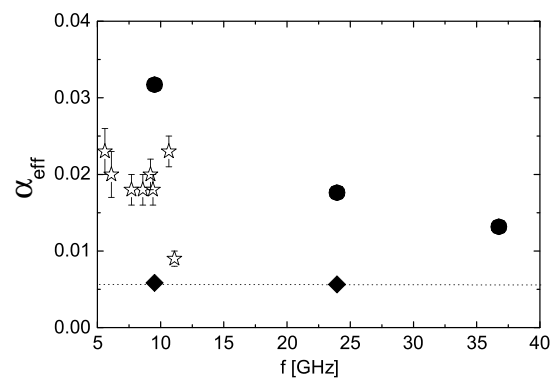

FIG. 5. Effective damping parameter as a function of the frequency for the $20 \mathrm{Au} / 20 \mathrm{Cr} / 16 \mathrm{Fe} / \mathrm{GaAs}$ sample. (O) was obtained using FMR [8] and (₹) are the results of TRMOKE measurements. In these experiments the applied field was parallel to the $[110]_{\mathrm{Fe}}$ direction. The $(\diamond)$ symbols correspond to FMR measurements in the perpendicular configuration and represent the Gilbert damping. rameters are shown in Fig. 4. The frequency independent damping parameter $\alpha$ in $\mathrm{Au} / \mathrm{Fe} / \mathrm{GaAs}$ and $\mathrm{Au} / \mathrm{Pd} / \mathrm{Fe} / \mathrm{GaAs}$ is described by Gilbert damping. The magnitude of the damping in the $20 \mathrm{Au} / 16 \mathrm{Fe} / \mathrm{GaAs}$ sample determined by TRMOKE was found to be $15 \%$ smaller than that found by a standard FMR technique with the same sample: $\quad \alpha_{\text {TRMOKE }}=3.2 \pm 0.3 \times 10^{-3} ; \quad \alpha_{\mathrm{FMR}}=$ $3.8 \pm 0.2 \times 10^{-3}$. The larger damping constant determined from FMR is probably a consequence of a small density of magnetic defects which lead to line broadening since in FMR the signal is averaged over millimeter sized samples. The additional damping due to spin pumping in the $20 \mathrm{Au} / 25 \mathrm{Pd} / 16 \mathrm{Fe} / \mathrm{GaAs}$ sample was found to be $\alpha_{\text {TRMOKE }}^{\text {add } 25 \mathrm{Pd}}=3.0 \pm 0.2 \times 10^{-3}$ in excellent agreement with that found by using standard FMR; $\alpha_{\mathrm{FMR}}^{\text {add }} 25 \mathrm{Pd}=2.9 \pm$ $0.2 \times 10^{-3}$ [7].

The dependence of the precessional frequency on $H_{B}$ was used to determine the $g$ factor and $4 \pi M_{\text {eff }}$. For the $20 \mathrm{Au} / 16 \mathrm{Fe} / \mathrm{GaAs}$ sample, one obtains $g=2.10 \pm 0.02$ and $4 \pi M_{\text {eff }}=17.0 \pm 0.2 \mathrm{kG}$ in agreement with FMR [4].

In ultrathin $\mathrm{Fe}$ layers covered by $\mathrm{Cr}(001)$ the relaxation was strongly affected by two-magnon scattering [8]. In two-magnon scattering the resonant FMR mode (usually $k=0$ ) scatters into other magnons having the same frequency. This mechanism is operative in the presence of magnetic defects where magnon momentum conservation is not required $[9,10]$. Magnetic films that are in contact with an antiferromagnetic layer often show large twomagon scattering due to spatially inhomogeneous exchange bias [11-13]. In the case of $\mathrm{Cr}$ on $\mathrm{Fe}(001)$, these defects are caused by step induced magnetic frustration between the magnetic moment of Fe and the spin density waves in $\mathrm{Cr}$ [14]. In the corresponding TRMOKE mea-

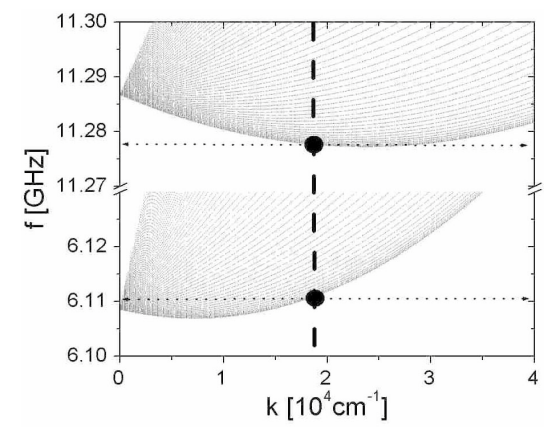

FIG. 6. Spin wave dispersion band (spin wave manifold) for a $16 \mathrm{ML}$ Fe film calculated for $H_{B}=0(f=6.10 \mathrm{GHz})$ and $H_{B}=$ $750 \mathrm{Oe}(f=11.29 \mathrm{GH} z)$ corresponding to the measurements shown in Figs. 3(a) and 3(d). The family of curves in the spin wave manifold is obtained using different angles between the magnetization and the spin wave ( $\boldsymbol{M}$ and $\boldsymbol{k}$ ). The bottom of the spin wave band corresponds to $\boldsymbol{k} \| \boldsymbol{M}$. The dashed vertical line represents the $k$ vector of the initial excitation of the magnetization $\sim \pi / 2 R$ and the symbols highlight the position of this excitation within the spin wave band. Note that at $f=6.1 \mathrm{GHz}$ many degenerate spin waves are available for scattering (horizontal arrow), while at $11.3 \mathrm{GHz}$ their number is substantially reduced. 
surements, the relaxation parameter becomes strongly frequency dependent due to two-magnon scattering, while for Gilbert damping there is no frequency dependence (cf. Figs. 4 and 5). Compared to rf techniques [11-13], the inhomogeneous excitation of the magnetization created by the Schottky diode switch has to be considered to understand why the relaxation depends so critically on the applied bias field, as shown in Fig. 3. In this experimental configuration, changing the applied magnetic dc field allows one to show the connection between the strength of magnon scattering and the available $k$ space of degenerate spin waves.

Equation (1) and Fig. 1 imply that the spatially most inhomogeneous Fourier component of the excitation field pulse is parallel to $\boldsymbol{M}$, and the magnetic excitation can be described by a wave vector with $k=2 \pi /(4 R)$. In Fig. 6 the spin wave dispersion spectra for in-plane $\boldsymbol{k}$ vectors are calculated from Eq. (10) in Ref. [15] for $f=6.1$ and $11.3 \mathrm{GHz}$ with magnetic parameters corresponding to the $16 \mathrm{ML}$ Fe film. Based on this figure one can understand why the strength of the two-magnon scattering critically depends on the bias field and the diameter of the laser pump beam focal spot. At $11.3 \mathrm{GHz}$ the excited mode has a $k$ vector close to the bottom of the spin wave manifold. In this case, there are obviously fewer degenerate magnons available compared to the lower bias fields where the initial excitation can find many degenerate spin waves, as illustrated by the intersection of the horizontal dotted line with the spin wave manifold in Fig. 6. This explains the decreased relaxation observed in Figs. 3(d) and 5. Hence, the Schottky diode configuration allows one to tune the strength of two-magnon scattering by means of the external magnetic bias field. The presence of out-of-phase oscillations in Fig. 3(b) is a consequence of mode-mode coupling in two-magnon scattering. A significant amount of the initial excitation scatters into spin wave modes which are out of phase with the initial excitation at the probed position. The use of a local probe reveals the mode-mode coupling in two-magnon scattering.

In Fig. 5 the relaxation corresponding to this series of TRMOKE measurements was put into context with the FMR measurements on the same sample [8] (the FMR linewidths were expressed by an effective damping parameter: $\alpha_{\text {eff }}=\Delta H \frac{\gamma}{\omega}$ ). The effective damping in the TRMOKE measurements was significantly smaller than that obtained from FMR at the corresponding frequency. This is expected since in our TRMOKE measurements a significant part of the magnon scattering is from large into small $k$ vectors (see Fig. 6). This contribution to the relaxation is largely ineffective when the magnetization is probed locally. It is striking that the intrinsic damping in $\mathrm{Fe} / \mathrm{Cr}$ samples (see data points for perpendicular FMR in Fig. 5) is very close to that found in $\mathrm{Pd} / \mathrm{Fe}$ and $\mathrm{Fe} / \mathrm{Au} / \mathrm{Fe}$ structures with the same $\mathrm{Fe}$ thickness, and enhanced compared to $\mathrm{Au} / \mathrm{Fe}$ samples. In $\mathrm{Pd} / \mathrm{Fe}$ samples the spin-sink effect is caused by the presence of paramagnons in Pd which lead to decoherence of the injected spin current [7]. In $\mathrm{Fe} / \mathrm{Au} / \mathrm{Fe}$ samples a perfect spin sink is provided by the second Fe layer [16]. This implies that the spin density wave in $\mathrm{Cr}$ also acts as a spin sink. The results of a recent FMR study of sputtered Permalloy films with $\mathrm{Cr}$ and Pd capping layers further support this conclusion [17].

Summary. - We have shown that our unique TRMOKE configuration allows one to excite resonant spin waves with a wavelength corresponding to twice the pump beam diameter. The damping constant determined by TRMOKE in $\mathrm{Fe} / \mathrm{GaAs}$ films capped by $\mathrm{Au}$ and $\mathrm{Pd}$ was found equal to that in standard FMR $(k \sim 0)$, indicating that the magnitude of the damping is independent of the lateral scale of the measurement and the excited $k$ vector for Gilbert damping. In contrast to this behavior, we demonstrated that for nonzero $k$ vectors the strength of two-magnon scattering rapidly changes with the applied bias field. In fact, the effective damping can be changed by as much as a factor of 2 by a slight tuning of the external field. This effect is a consequence of the field induced shift of the bottom of the spin wave band with respect to the $k$ vector of the excited spin waves. When the bottom of the band and the $k$ vector of the excited spin waves coincide, the magnon degeneracy is lifted and two-magnon processes are forbidden.

Financial support from NSERC and CIAR (Canada) is gratefully acknowledged. This work was supported by the DFG Priority Program No. SPP 1133 (Germany).

[1] N. Smith et al., J. Appl. Phys. 91, 7454 (2002).

[2] R. D. McMichael et al., Phys. Rev. Lett. 90, 227601 (2003).

[3] Y. Acremann et al., Nature (London) 414, 51 (2001).

[4] R. Urban, G. Woltersdorf, and B. Heinrich, Phys. Rev. Lett. 87, 217204 (2001).

[5] G. Woltersdorf and B. Heinrich, Phys. Rev. B 69, 184417 (2004).

[6] B. Heinrich and J. F. Cochran, Adv. Phys. 42, 523 (1993).

[7] J. Foros et al., J. Appl. Phys. 97, 10A714 (2005).

[8] R. Urban et al., Phys. Rev. B 65, 020402 (2002).

[9] M. Sparks, Ferromagnetic Relaxation Theory (Mc-GrawHill, New York, 1966).

[10] C. E. Patton and F. Humphrey, J. Appl. Phys. 37, 4269 (1966).

[11] R. D. McMichael et al., J. Appl. Phys. 83, 7037 (1998).

[12] C. Mathieu et al., J. Appl. Phys. 83, 2863 (1998).

[13] S. M. Rezende et al., Phys. Rev. B 63, 214418 (2001).

[14] B. Heinrich et al., Phys. Rev. B 59, 14520 (1999).

[15] R. Arias and D. L. Mills, Phys. Rev. B 60, 7395 (1999).

[16] B. Heinrich et al., Phys. Rev. Lett. 90, 187601 (2003).

[17] R. C. Oliveira et al., J. Magn. Magn. Mater. 272, e795 (2004). 